\title{
Propagation of magneto-thermo surface waves in non-homogenous viscoelastic medium of higher order under gravity and couple-stress
}

\author{
Rajneesh Kakar ${ }^{1, *}$, Shikha Kakar ${ }^{2}$ \\ ${ }^{1}$ Principal, DIPS Polytechnic College, Hoshiarpur, India \\ ${ }^{2}$ Faculty of Electrical Engineering, SBBSIET Padhiana Jalandhar, India
}

Email address:

rkakar_163@rediffmail.com (R. Kakar)

\section{To cite this article:}

Rajneesh Kakar, Shikha Kakar. Propagation of Magneto-Thermo Surface Waves in Non-Homogenous Viscoelastic Medium of Higher Order under Gravity and Couple-Stress, International Journal of Materials Science and Applications. Vol. 2, No. 1, 2013, pp. 8-19. doi: $10.11648 /$ j.ijmsa.20130201.12

\begin{abstract}
The present study reveals the effects of various parameters and non-homogeneity on the surface waves propagating in viscoelastic medium. The theory of generalized surface waves has firstly been developed and then it has been employed to study the surface waves. Dispersion relation for Stoneley waves, Rayleigh waves and Love waves has been deduced. It has been observed that in the absence of viscosity, temperature, gravity, magnetism, couple stress and nonhomogeneity of the material medium, the results obtained are in well agreement with the corresponding classical results.
\end{abstract}

Keywords: Viscoelastic Solid Medium, Gravity, Couple Stress, Inhomogeneities, Variable Density

\section{Introduction}

When seismic waves propagate underground, they are influenced not only by the anisotropy of the media, but also by intrinsic viscosity of media given by Carcione [1]. Therefore, in order to accurately describe the underground propagation of the seismic waves and then more precisely guide seismic data acquisition, processing and interpretation, media models should be chosen that can simultaneously imitate anisotropic characteristics of formation and viscoelastic characteristics for numerical simulation and analysis of wave fields As a result, the theory of surface waves has been developed by Stoneley [2], Bullen [3], Ewing et. al. [4], Hunters and Jeffreys [5].

The effect of gravity on wave propagation in an elastic solid medium was first considered by Bromwich [6], treating the force of gravity as a type of body force. Love [7] extended the work of Bromwich investigated the influence of gravity on superfacial waves and showed that the Rayleigh wave velocity is affected by the gravity field. Sezawa [8] studied the dispersion of elastic waves propagated on curved surfaces.

The transmission of elastic waves through a stratified solid medium was studied by Thomson [9]. Haskell [10] studied the dispersion of surface waves in multilayered media. A source on elastic waves is the monograph of Ewing, Jardtezky and Press [11]. Biot [12] studied the influence of gravity on Rayleigh waves, assuming the force of gravity to create a type of initial stress of hydrostatic nature and the medium to be incompressible. Taking into account, the effect of initial stresses and using Biot's theory of incremental deformations, Dey modified the work of Jones [13]. De and Sengupta [14] studied many problems of elastic waves and vibrations under the influence of gravity field. Sengupta and Acharya [15] studied the influence of gravity on the propagation of waves in a thermoelastic layer. Brunelle [16] studied the surface wave propagation under initial tension of compression. Wave propagation in a thin two-layered laminated medium with stress couples under initial stresses was studied by Roy [17]. Datta [18] studied the effect of gravity on Rayleigh wave propagation in a homogeneous, isotropic elastic solid medium. Goda [19] studied the effect of inhomongeneity and anisotropy on Stoneley waves. Recently Abd-Alla and Ahmed [20] studied the Rayleigh waves in an orthotropic thermoelastic medium under gravity field and initial stress.

Recently, Kakar et al. [21-25] investigated various surface waves in non homogeneous viscoelastic media of higher order under gravity. However, the effect of couple stress on surface waves has not been discussed so far, therefore authors have solved the problem of $\mathrm{n}^{\text {th }}$ order viscoelas- 
tic surface waves under gravity and couple stress involving time rate of strain. In this study, it is assumed that the surface waves are propagating in isotropic, non-homogeneous and viscoelastic medium under the effect of various parameters such as temperature, magnetic field, gravity and couple stress. The dispersion relations are obtained for Stoneley, Rayleigh and Love waves by using Biot's theory of incremental deformations. has been used to obtain the wave velocity equation. Further these equations are in complete agreement with the corresponding classical results in the absence of various inhomogeneities of the material medium.

\section{Formulation of the Problem}

Let $\mathrm{M}_{1}$ and $\mathrm{M}_{2}$ be two non-homogeneous, viscoelastic, isotropic, semi-finite media (Fig.1). They are perfectly welded in-contact to prevent any relative motion or sliding before and after the disturbances and that the continuity of displacement, stress etc. hold good across the common boundary surface. Further the mechanical properties of $\mathrm{M}_{1}$ are different from those of $\mathrm{M}_{2}$. These media extend to an infinite great distance from the origin and are separated by a plane horizontal boundary and $\mathrm{M}_{2}$ is to be taken above $\mathrm{M}_{1}$.

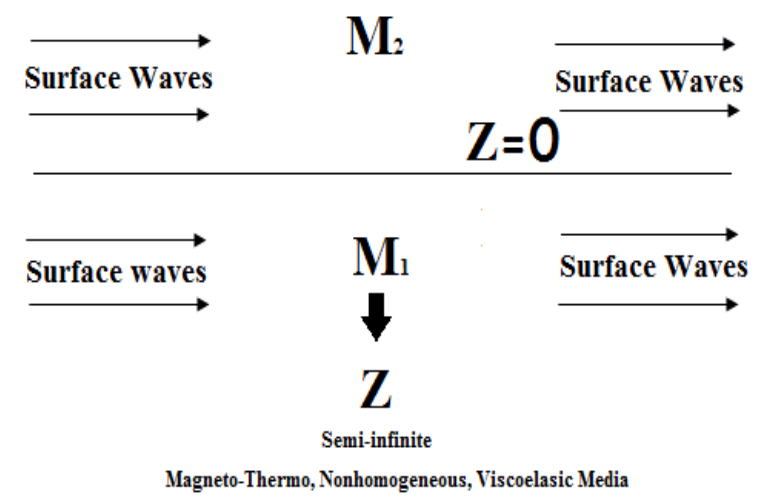

Fig.1. Geometry of the problem.

Let Oxyz be a set of orthogonal Cartesian co-ordinates and let $\mathrm{O}$ be the any point on the plane boundary and $\mathrm{Oz}$ points vertically downward to the medium $\mathrm{M}_{1}$. We consider the possibility of a type of wave traveling in the direction $\mathrm{Ox}$, in such a manner that the disturbance is largely confined to the neighborhood of the boundary which implies that wave is a surface wave.

It is assume that at any instant, all particles in any line parallel to Oy having equal displacement and all partial derivatives with respect to y are zero. Further let us assume that $\mathrm{u}, \mathrm{v}, \mathrm{w}$ is the components of displacements at any point $(\mathrm{x}, \mathrm{y}, \mathrm{z})$ at any time $\mathrm{t}$.

It is also assume that gravitational field produces a hydrostatic initial stress is produced by a slow process of creep where the shearing stresses tend to become small or vanish after a long period of time. The equilibrium conditions of initial stress are

$$
\frac{\partial \tau}{\partial x}=0, \frac{\partial \tau}{\partial z}+\rho g=0
$$

Also, [12]

$$
\begin{aligned}
& \tau_{11}=\tau_{33}=\tau \\
& \tau_{13}=0
\end{aligned}
$$

The dynamical equations of motion for threedimensional non-homogeneous, isotropic, viscoelastic solid medium in Cartesian co-ordinates with Eq. (1) are

$$
\begin{aligned}
& \frac{\partial \tau_{11}}{\partial x}+\frac{\partial \tau_{12}}{\partial y}+\frac{\partial \tau_{13}}{\partial z}+\rho g \frac{\partial w}{\partial x}=\rho \frac{\partial^{2} u}{\partial t^{2}} \\
& \frac{\partial \tau_{12}}{\partial x}+\frac{\partial \tau_{22}}{\partial y}+\frac{\partial \tau_{23}}{\partial z}+\rho g \frac{\partial w}{\partial y}=\rho \frac{\partial^{2} v}{\partial t^{2}} \\
& \frac{\partial \tau_{13}}{\partial x}+\frac{\partial \tau_{23}}{\partial y}+\frac{\partial \tau_{33}}{\partial z}-\rho g\left(\frac{\partial u}{\partial x}+\frac{\partial v}{\partial y}\right)=\rho \frac{\partial^{2} w}{\partial t^{2}}
\end{aligned}
$$

Where $\rho$ be the density of the material medium and $\tau_{i j}=\tau_{j i} \forall \mathrm{i}, \mathrm{j}$ are the stress components. Let us consider that the medium is a perfect electric conductor, we take the linearized Maxwell equations governing the electromagnetic field, taking into account absence of the displacement current (in system-international unit) in the form

$$
\begin{aligned}
& \vec{\nabla} \cdot \overrightarrow{\mathrm{E}}=0 \\
& \vec{\nabla} \cdot \overrightarrow{\mathrm{B}}=0 \\
& \vec{\nabla} \times \overrightarrow{\mathrm{E}}=-\frac{\bar{\partial} \overrightarrow{\mathrm{B}}}{\partial t} \\
& \vec{\nabla} \times \overrightarrow{\mathrm{B}}=\mu_{e} \varepsilon_{e} \frac{\bar{\partial} \overrightarrow{\mathrm{E}}}{\partial t} .
\end{aligned}
$$

Where, $\overrightarrow{\mathrm{E}}, \overrightarrow{\mathrm{B}}, \mu_{e}$ and $\varepsilon_{e}$ are electric field, magnetic field induction, permeability and permittivity of the medium.

The value of magnetic field intensity is

$$
\overrightarrow{\mathrm{H}}(0,0, \mathrm{H})=\overrightarrow{\mathrm{H}}_{0}+\overrightarrow{\mathrm{H}}_{i}
$$

We consider an orthotropic elastic solid under constant primary magnetic field $\overrightarrow{\mathrm{H}}$ acting on y-axis and $\overrightarrow{\mathrm{H}}_{i}$ is the perturbation in the magnetic field intensity.

It is assumed that prior to the existence of any disturbance both the media are everywhere at the constant absolute temperature $\mathrm{T}_{0}$.

The stress-strain relations for general isotropic, thermo, viscoelastic medium, according to Voigt are [26]

$$
\tau_{\mathrm{ij}}=2 \mathrm{D}_{\mu} \mathrm{e}_{\mathrm{ij}}+\left(\mathrm{D}_{\lambda} \Delta-\mathrm{D}_{\beta} \mathrm{T}+H_{0}^{2} \Delta D_{\mathrm{m}_{e}}\right) \delta_{\mathrm{ij}}
$$

where, 
$\triangle=\frac{\partial u}{\partial x}+\frac{\partial v}{\partial y}+\frac{\partial w}{\partial z}$ and $\mathrm{D}_{\lambda}, \mathrm{D}_{\mu}, \mathrm{D}_{\beta}$ are elastic constants.

Introducing Eq. (5) in Eq. (2a), Eq. (2b), Eq. (2c), we get

$$
\begin{aligned}
& D_{\lambda} \frac{\partial \Delta}{\partial x}+\Delta \frac{\partial D_{\lambda}}{\partial x}+ \\
& 2 D_{\mu} \frac{\partial^{2} u}{\partial x^{2}}+2 \frac{\partial u}{\partial x} \frac{\partial D_{\mu}}{\partial x}- \\
& D_{\beta} \frac{\partial T}{\partial x}+D_{\mu} \frac{\partial}{\partial z}\left[\frac{\partial u}{\partial z}+\frac{\partial w}{\partial x}\right] \\
& +\left[\frac{\partial u}{\partial z}+\frac{\partial w}{\partial x}\right] \frac{\partial D_{\mu}}{\partial z} \\
& H_{0}^{2} D_{\mathrm{m}_{e}} \frac{\partial \mathrm{D}}{\partial x}+H_{0}^{2} \Delta \frac{\partial D_{\mathrm{m}_{e}}}{\partial x} \\
& +\rho g \frac{\partial w}{\partial x}=\rho \frac{\partial^{2} u}{\partial t^{2}} \\
& D_{\mu} \nabla^{2} v+\frac{\partial v}{\partial x} \frac{\partial D_{\mu}}{\partial x}+\frac{\partial v}{\partial z} \frac{\partial D_{\mu}}{\partial z}=\rho \frac{\partial^{2} v}{\partial t^{2}} \\
& D_{\mu} \frac{\partial}{\partial x}\left(\frac{\partial u}{\partial z}+\frac{\partial w}{\partial x}\right)+\left(\frac{\partial u}{\partial z}+\frac{\partial w}{\partial x}\right) \frac{\partial D_{\mu}}{\partial x} \\
& +2 D_{\mu} \frac{\partial^{2} w}{\partial z^{2}}+2 \frac{\partial w}{\partial z} \frac{\partial D_{\mu}}{\partial z}+D_{\lambda} \frac{\partial \Delta}{\partial z} \\
& +\Delta \frac{\partial D_{\lambda}}{\partial z}-D_{\beta} \frac{\partial T}{\partial z}-T \frac{\partial D_{\beta}}{\partial z} \\
& H_{0}^{2} D_{\mathrm{m}_{e}} \frac{\partial \mathrm{D}}{\partial z}+H_{0}^{2} \Delta \frac{\partial D_{\mathrm{m}_{e}}}{\partial z}-\rho g \frac{\partial u}{\partial x}=\rho \frac{\partial^{2} w}{\partial t^{2}}
\end{aligned}
$$

We assume that the non-homogeneities for the media $M_{1}$ and $\mathrm{M}_{2}$ are given by

$$
\begin{gathered}
\mathrm{D}_{\lambda}=\sum_{K=0}^{n} \lambda_{K} e^{m z} \frac{\partial^{K}}{\partial t^{K}}, \\
\mathrm{D}_{\mu}=\sum_{K=0}^{n} \mu_{K} e^{m z} \frac{\partial^{K}}{\partial t^{K}}, \\
\mathrm{D}_{\beta}=\sum_{K=0}^{n} \beta_{K} e^{m z} \frac{\partial^{K}}{\partial t^{K}} \\
D_{\mathrm{m}_{e}}=\sum_{K=0}^{n}\left(\mu_{e}\right)_{K} e^{m z} \frac{\partial^{K}}{\partial t^{K}}, \\
D_{\eta}=\sum_{K=0}^{N} \eta_{K} e^{m z} \frac{\partial^{K}}{\partial t^{K}},
\end{gathered}
$$

$$
\rho=\rho_{0} e^{m z}
$$

and

$$
\begin{aligned}
& \mathrm{D}^{\prime} \lambda=\sum_{K=0}^{n} \lambda^{{ }_{K}} e^{\mathrm{lz} z} \frac{\partial^{K}}{\partial t^{K}} \\
& \mathrm{D} \mu=\sum_{K=0}^{n} \mu^{\prime}{ }_{K} e^{\mathrm{lz} z} \frac{\partial^{K}}{\partial t^{K}} \\
& \mathrm{D} \beta=\sum_{K=0}^{n} \beta_{K} e^{\mathrm{lz} z} \frac{\partial^{K}}{\partial t^{K}} \\
& D_{\mu_{e}}^{\prime}=\sum_{K=0}^{n}\left(\mu^{\prime}\right)_{K} e^{m z} \frac{\partial^{K}}{\partial t^{K}} \\
& D^{\prime}{ }_{\eta}=\sum_{K=0}^{N} \eta^{\prime}{ }_{K} e^{l z} \frac{\partial^{K}}{\partial t^{K}} \\
& \rho^{\prime}=\rho^{\prime}{ }_{0}^{l z} e^{l z}
\end{aligned}
$$

where $\lambda_{0}, \mathrm{M}_{0}, \lambda_{0}^{\prime}, \mu_{0}^{\prime}$ are elastic constants, whereas $\beta_{0}$, $\beta_{0}^{\prime}$ are thermal parameters are $\rho_{0}, \rho_{0}^{\prime}, m, n$ are constants. $\lambda_{\mathrm{K}}, \mu_{\mathrm{K}}(\mathrm{K}=0,1,2, \ldots \mathrm{n})$ are the parameters associated with $\mathrm{K}^{\text {th }}$ order viscoelasticity and $\beta_{\mathrm{K}}$ and $\left(\mu_{\mathrm{e}}\right)_{\mathrm{K}}(\mathrm{K}=1,2, \ldots ., \mathrm{n})$ are the thermal and magnetic parameters associated with $\mathrm{K}^{\text {th }}$ order. $\mathrm{T}$ is the absolute temperature over the initial temperature $\mathrm{T}_{0}$.

Due to temperature rise of the material medium, it has been observed that all the parameters representing elastic property, the effect of viscosity and thermal field depends on the temperature and ultimately depends on time $t$. In a thermo viscoelastic solid, the thermal parameters $\beta_{K}(K=0$, $1, \ldots \ldots n)$ are given by $\beta_{K}=\left(3 \lambda_{K}+2 \mu_{K}\right) \alpha_{t}$, where $\alpha_{t}$ be the coefficient of linear expansion of solid.

$$
\begin{gathered}
\left(G_{\lambda}+G_{\mu}+H_{0}^{2} G_{\mathrm{m}_{e}}\right) \frac{\partial \Delta}{\partial x}+G_{\mu} \nabla^{2} u \\
+m G_{\mu}\left(\frac{\partial u}{\partial z}+\frac{\partial w}{\partial x}\right)+m \nabla^{2}\left(\frac{\partial^{2} w}{\partial x \partial z}-\frac{\partial^{2} u}{\partial z^{2}}\right) G_{\eta} \\
-G_{\beta} \frac{\partial T}{\partial x}+\rho g \frac{\partial w}{\partial x}=\rho_{0} \frac{\partial^{2} u}{\partial t^{2}} \\
G_{\mu} \nabla^{2} \mathrm{v}+G_{\mu} m \frac{\partial v}{\partial z}=\rho_{0} \frac{\partial^{2} v}{\partial t^{2}} \\
\left(G_{\lambda}+G_{\mu}+H_{0}^{2} G_{\mathrm{m}_{e}}\right) \frac{\partial \Delta}{\partial z}+\Delta G_{\lambda} m \\
+2 G_{\mu} m+2 G_{\mu} m \frac{\partial w}{\partial z}+m \nabla^{2}\left(\frac{\partial^{2} w}{\partial x \partial z}-\frac{\partial^{2} u}{\partial z^{2}}\right) G_{\eta} \\
-m G_{\beta} T m H_{0}^{2} \Delta G_{\mathrm{m}_{e}}-\rho g \frac{\partial u}{\partial x}=\rho_{0} \frac{\partial^{2} w}{\partial t^{2}}
\end{gathered}
$$

where, 


$$
\begin{gathered}
\mathrm{G}_{\lambda}=\sum_{K=0}^{n} \lambda_{K} \frac{\partial^{K}}{\partial t^{K}}, \\
\mathrm{G}_{\mu}=\sum_{K=0}^{n} \mu_{K} \frac{\partial^{K}}{\partial t^{K}}, \\
\mathrm{G}_{\beta}=\sum_{K=0}^{n} \beta_{K} \frac{\partial^{K}}{\partial t^{K}} \\
G_{\mu_{e}}=\sum_{K=0}^{n}\left(\mu_{e}\right)_{K} \frac{\partial^{K}}{\partial t^{K}}, \\
\nabla^{2}=\frac{\partial^{2}}{\partial x^{2}}+\frac{\partial^{2}}{\partial z^{2}}, \\
\Delta=\frac{\partial u}{\partial x}+\frac{\partial v}{\partial y}+\frac{\partial w}{\partial z}
\end{gathered}
$$

To investigate the surface wave propagation along the direction of $\mathrm{Ox}$, we introduce displacement potential $\phi(\mathrm{x}, \mathrm{z}, \mathrm{t})$ and $\psi(\mathrm{x}, \mathrm{z}, \mathrm{t})$ which are related to the displacement components as follows:

$$
\begin{gathered}
\mathrm{u}=\frac{\partial \phi}{\partial x}-\frac{\partial \psi}{\partial z} \\
\mathrm{w}=\frac{\partial \phi}{\partial z}+\frac{\partial \psi}{\partial x}
\end{gathered}
$$

The displacement potential $\phi(\mathrm{x}, \mathrm{z}, \mathrm{t})$ and $\psi(\mathrm{x}, \mathrm{z}, \mathrm{t})$ in Eq. (10) satisfy the following Laplace equation (known as dilation and rotation and are associated with $\mathrm{P}$ and $\mathrm{SV}$ waves)

$$
\begin{aligned}
& \nabla^{2} \phi=\frac{\partial u}{\partial x}+\frac{\partial w}{\partial z}=\Delta \\
& \nabla^{2} \psi=\frac{\partial w}{\partial x}-\frac{\partial u}{\partial z}=2 \Omega
\end{aligned}
$$

Substituting Eq. (10) in Eqs (8a), (8b) and (8c), we get

$$
\begin{gathered}
\mathrm{G}_{\mathrm{R}} \nabla^{2} \phi+\mathrm{mG}_{\mathrm{S}}\left(2 \frac{\partial \phi}{\partial z}+\frac{\partial \psi}{\partial x}\right) \\
-\mathrm{GLT}+g \frac{\partial \psi}{\partial x}=\frac{\partial^{2} \phi}{\partial t^{2}} \\
\mathrm{G}_{\mathrm{S}} \nabla^{2} \mathrm{v}+\mathrm{mG}_{\mathrm{S}} \frac{\partial v}{\partial z}=\frac{\partial^{2} v}{\partial t^{2}} \\
\mathrm{G}_{\mathrm{S}} \nabla^{2} \psi+\mathrm{mG}_{\mathrm{P}} \frac{\partial \phi}{\partial x}+
\end{gathered}
$$

\section{Solution of the Problem} following forms.
Now our main objective to solve Eq. (11a), Eq. (11b), Eq.
(11c) and Eq. (13), for this, we seek the solutions in the

Now our main objective to solve Eq. (11a), Eq. (11b), Eq.
(11c) and Eq. (13), for this, we seek the solutions in the

$$
\begin{gathered}
U_{K R}^{2}=\frac{\lambda_{K}+2 \mu_{K}+H_{0}^{2}\left(\mathrm{~m}_{e}\right)_{K}}{\rho_{0}}, \\
U_{K S}^{2}=\frac{\mu_{K}}{\rho_{0}}, \\
U_{K P}^{2}=\frac{\lambda_{K}+H_{0}^{2}\left(\mathrm{~m}_{e}\right)_{K}}{\rho_{0}}, \\
U_{K L}^{2}=\frac{\beta_{K}}{\rho_{0}}
\end{gathered}
$$

and

$$
\begin{aligned}
& \mathrm{G}_{\mathrm{R}}=\sum_{K=0}^{n} U_{K R}^{2} \frac{\partial^{K}}{\partial t^{K}}, \\
& \mathrm{G}_{\mathrm{S}}=\sum_{K=0}^{n} U_{K S}^{2} \frac{\partial^{K}}{\partial t^{K}}, \\
& \mathrm{G}_{\mathrm{P}}=\sum_{K=0}^{n} U_{K P}^{2} \frac{\partial^{K}}{\partial t^{K}}, \\
& \mathrm{G}_{\mathrm{L}}=\sum_{K=0}^{n} U_{K L}^{2} \frac{\partial^{K}}{\partial t^{K}}, \\
& G_{q}=\sum_{K=0}^{n} U_{K q}^{2} \frac{\partial^{K}}{\partial t^{K}}
\end{aligned}
$$

To determine T, Fourier's law of heat conduction

$$
\mathrm{p} \nabla^{2} \mathrm{~T}=C_{v} \frac{\partial T}{\partial t}+T_{0} G_{L} \frac{\partial}{\partial t}\left(\nabla^{2} \phi\right)
$$

where $\mathrm{K}$ be the thermal conductivity and obeys the law as given by $\mathrm{K}=\mathrm{K} 0 \mathrm{emz}, \mathrm{p}=\frac{K_{0}}{\rho_{0}}$ and $\mathrm{C}$ be the specific heat of the body at constant volume.

Further, similar relations in medium $\mathrm{M}_{2}$ can be found out by replacing $\lambda_{\mathrm{K}}, \mu_{\mathrm{K}}, \beta_{\mathrm{K}}, \rho_{0}$ by $\lambda_{\mathrm{K}}^{\prime}, \mu_{\mathrm{K}}^{\prime}, \beta_{\mathrm{K}}^{\prime}, \rho_{0}^{\prime}$ and so on. 
$(\phi, \psi, \mathrm{T}, \mathrm{v})=\left[\mathrm{f}(\mathrm{z}), \mathrm{V}(\mathrm{z}), \mathrm{T}_{1}(\mathrm{z}), \mathrm{h}(\mathrm{z})\right] \mathrm{e}^{\mathrm{i} \alpha(\mathrm{x}-\mathrm{ct})}$

Using Eq. (12) in Eq. (9a), Eq. (9b), Eq. (9c) and Eq. (11), we get a set of differential equations for the medium $\mathrm{M}_{1}$ as follows:

$$
\begin{aligned}
& \frac{d^{2} f}{d z^{2}}+2 m f_{1}^{2} \frac{d f}{d z}+h_{1}{ }^{2} f \\
& +\left(i \alpha m f_{1}^{2}+i \alpha g\right) h-g_{1}{ }^{2} T_{1}=0 \\
& \frac{d^{2} V}{d z^{2}}+m \frac{d V}{d z}+D_{1}{ }^{2} V=0 \\
& \frac{d^{4} h}{d z^{4}}+C_{1}^{2} \frac{d^{2} h}{d z^{2}}+2 E_{1}^{2} \frac{d h}{d z} \\
& +F_{1}^{2} h+\left(i \alpha m l_{1}^{2}-i \alpha g\right) f=0 \\
& \frac{d^{2} T_{1}}{d z^{2}}+A T_{1}+B\left(\frac{d^{2} f}{d z^{2}}-\alpha^{2} f\right)=0
\end{aligned}
$$

where,

$$
\begin{gathered}
\mathrm{f}_{1} 2=\frac{\sum_{K=0}^{n} U_{K S}^{2}(-i \alpha c)^{K}}{\sum_{K=0}^{n} U_{K R}^{2}(-i \alpha c)^{K}}, \\
\mathrm{~h}_{1}^{2}=\frac{\alpha^{2} c^{2}}{\sum_{K=0}^{n} U_{K R}^{2}(-i \alpha c)^{K}}-\alpha^{2}, \\
C_{1}^{2}=-\left(2 \alpha^{2}+d_{1}^{2}\right), \\
d_{1}^{2}=\frac{G_{s}}{G_{q}}+\frac{\sum_{K=0}^{n} U_{K S}^{2}(-i \alpha c)^{K}}{\sum_{K=0}^{n} U_{K q}^{2}(-i \alpha c)^{K}}
\end{gathered}
$$

(couple-stress parameter).

$$
\begin{gathered}
\mathrm{D}_{1}{ }^{2}=\frac{\alpha^{2} c^{2}}{\sum_{K=0}^{n} U_{K S}^{2}(-i \alpha c)^{K}}-\alpha^{2}, \\
l_{1} 2=\frac{\sum_{K=0}^{n} U_{K P}^{2}(-i \alpha c)^{K}}{\sum_{K=0}^{n} U_{K S}^{2}(-i \alpha c)^{K}}, \\
\mathrm{~g}_{1} 2=\frac{\sum_{K=0}^{n} U_{K L}^{2}(-i \alpha c)^{K}}{\sum_{K=0}^{n} U_{K R}^{2}(-i \alpha c)^{K}}
\end{gathered}
$$

$$
\begin{aligned}
& E_{1}^{2}=-2 l d_{1}^{2}, \\
& F_{1}^{2}=\alpha^{2} l^{2}+\alpha^{4}-\frac{\alpha^{2} c^{2}}{G q}, \\
& \mathrm{~A}=\frac{C_{v} i \alpha c}{p}-\alpha^{2}, \mathrm{~B}=\frac{i \alpha c T_{0}}{p} G_{L}
\end{aligned}
$$

and those for the medium $\mathrm{M}_{2}$ are given by

$$
\begin{aligned}
& \frac{d^{2} f^{\prime}}{d z^{2}}+2 m f_{1}^{\prime 2} \frac{d f}{d z}+h_{1}^{2} f^{\prime} \\
& +\left(i \alpha m f_{1}^{\prime 2}+i \alpha g\right) h-g_{1}^{\prime 2} T_{1}=0, \\
& \frac{d^{2} V^{\prime}}{d z^{2}}+m \frac{d V^{\prime}}{d z}+D_{1}^{\prime 2} V^{\prime}=0, \\
& \frac{d^{4} h^{\prime}}{d z^{4}}+C_{1}^{\prime 2} \frac{d^{2} h^{\prime}}{d z^{2}}+2 E_{1}^{\prime 2} \frac{d h^{\prime}}{d z} \\
& +F_{1}^{\prime 2} h+\left(i \alpha m l_{1}^{\prime 2}-i \alpha g\right) f^{\prime}=0, \\
& \frac{d^{2} T_{1}}{d z^{2}}+A^{\prime} T_{1}+B^{\prime}\left(\frac{d^{2} f^{\prime}}{d z^{2}}-\alpha^{2} f^{\prime}\right)=0 .
\end{aligned}
$$

where,

$$
\begin{gathered}
f_{1}^{\prime 2}=\frac{\sum_{K=0}^{n} U_{K S}^{\prime 2}(-i \alpha c)^{K}}{\sum_{K=0}^{n} U_{K R}^{\prime 2}(-i \alpha c)^{K}}, \\
\mathrm{~h}_{1}^{\prime 2}=\frac{\alpha^{2} c^{2}}{\sum_{K=0}^{n} U_{K R}^{\prime 2}(-i \alpha c)^{K}}-\alpha^{2}, \\
C_{1}^{\prime 2}=-\left(2 \alpha^{2}+d_{1}^{2}\right), \\
d_{1}^{\prime 2}=\frac{G_{s}}{G_{q}}+\frac{\sum_{K=0}^{n} U_{K S}^{\prime 2}(-i \alpha c)^{K}}{\sum_{K=0}^{n} U_{K q}^{\prime 2}(-i \alpha c)^{K}},
\end{gathered}
$$

(couple-stress parameter).

$$
\begin{aligned}
E_{1}^{\prime 2} & =-2 l d_{1}^{\prime 2}, \\
F_{1}^{\prime 2} & =\alpha^{2} l^{2}+\alpha^{4}-\frac{\alpha^{2} c^{2}}{G^{\prime} q}, \\
\mathrm{D}_{1}^{\prime 2} & =\frac{\alpha^{2} c^{2}}{\sum_{K=0}^{n} U_{K S}^{\prime 2}(-i \alpha c)^{K}}-\alpha^{2},
\end{aligned}
$$




$$
\begin{gathered}
l_{1}{ }^{2}=\frac{\sum_{K=0}^{n} U_{K P}^{\prime 2}(-i \alpha c)^{K}}{\sum_{K=0}^{n} U_{K S}^{\prime 2}(-i \alpha c)^{K}}, \\
\mathrm{~g}_{1}{ }^{2}=\frac{\sum_{K=0}^{n} U_{K L}^{\prime 2}(-i \alpha c)^{K}}{\sum_{K=0}^{n} U_{K R}^{\prime 2}(-i \alpha c)^{K}}, \\
\mathrm{~A}^{\prime}=\frac{C_{v} i \alpha c}{p}-\alpha^{2}, \mathrm{~B}^{\prime}=\frac{i \alpha c T_{0}}{p^{\prime}} G_{L}^{\prime}
\end{gathered}
$$

Eq. (15) and Eq. (17) must have exponential solutions in order that $f, j, T_{1}$, h will describe surface waves, and they must become varnishing small as $\mathrm{z} \rightarrow \infty$.

Hence for the medium $\mathrm{M}_{1}$

$$
\begin{aligned}
& \phi(\mathrm{x}, \mathrm{z}, \mathrm{t})= \\
& \left\{A_{1} e^{-\lambda_{1} z}+B_{1} e^{-\lambda_{2} z}+C_{1} e^{-\lambda_{3} z}+D_{1} e^{-\lambda_{4} z}\right\} e^{i \alpha(x-c t)} \\
& \psi(\mathrm{x}, \mathrm{z}, \mathrm{t})= \\
& \left\{A_{2} e^{-\lambda_{1} z}+B_{2} e^{-\lambda_{2} z}+C_{2} e^{-\lambda_{3} z}+D_{2} e^{-\lambda_{4} z}\right\} e^{i \alpha(x-c t)} \\
& \lambda^{8}+\xi_{1} \lambda^{7}+\xi_{2} \lambda^{6}+\xi_{3} \lambda^{5}+\xi_{4} \lambda^{4}+\xi_{5} \lambda^{3}+\xi_{6} \lambda^{2}+\xi_{7} \lambda+\xi_{8}=0, \\
& \mathrm{v}(\mathrm{x}, \mathrm{z}, \mathrm{t})=E^{\prime} e^{-\lambda_{4}^{\prime} z+i \alpha(x-c t)}
\end{aligned}
$$

$$
\begin{gathered}
\mathrm{T}(\mathrm{x}, \mathrm{z}, \mathrm{t})= \\
\left\{A_{3} e^{-\lambda_{1} z}+B_{3} e^{-\lambda_{2} z}+C_{3} e^{-\lambda_{3} z}+D_{3} e^{-\lambda_{4} z}\right\} e^{i \alpha(x-c t)} \\
\mathrm{v}(\mathrm{x}, \mathrm{z}, \mathrm{t})=E e^{-\lambda_{4} z+i \alpha(x-c t)}
\end{gathered}
$$

For finite disturbances as $\mathrm{z} \rightarrow \infty$.for medium $\mathrm{M}_{1}$ must hold $\operatorname{Re}\left(\lambda_{i}\right)>0$ for $i=1,2,3,4,5$.

Similarly for the medium $\mathrm{M}_{2}$ are given by

$$
\begin{gathered}
\phi(\mathrm{x}, \mathrm{z}, \mathrm{t})= \\
\left\{A_{1}^{\prime} e^{-\lambda_{1}^{\prime} z}+B^{\prime}{ }_{1} e^{-\lambda_{2} z}+C^{\prime}{ }_{1} e^{-\lambda_{3}^{\prime} z}+D^{\prime}{ }_{1} e^{-\lambda_{4}^{\prime} z}\right\} e^{i \alpha(x-c t)} \\
\psi(\mathrm{x}, \mathrm{z}, \mathrm{t})= \\
\left\{A^{\prime}{ }_{2} e^{-\lambda_{1}^{\prime} z}+B^{\prime}{ }_{2} e^{-\lambda_{2}^{\prime} z}+C^{\prime}{ }_{2} e^{-\lambda_{3}^{\prime} z}+D^{\prime}{ }_{2} e^{-\lambda_{4}^{\prime} z}\right\} e^{i \alpha(x-c t)}
\end{gathered}
$$$$
\mathrm{T}(\mathrm{x}, \mathrm{z}, \mathrm{t})=
$$$$
\left\{A^{\prime}{ }_{3} e^{-\lambda_{1}^{\prime} z}+B^{\prime}{ }_{3} e^{-\lambda_{2}^{\prime} z}+C^{\prime}{ }_{3} e^{-\lambda_{3}^{\prime} z}+D^{\prime}{ }_{3} e^{-\lambda_{4}^{\prime} z}\right\} e^{i \alpha(x-c t)}
$$

For finite disturbances as $\mathrm{z} \rightarrow-\infty$.for medium $\mathrm{M}_{2}$ must

Where $\lambda_{j}$ and $\lambda_{j}^{\prime}(j=1,2,3,4)$ are the real roots of the

where,

$$
\begin{aligned}
& \xi_{1}=2 \mathrm{mf}_{1}^{2}, \xi_{2}=\mathrm{A}+\mathrm{Bg}_{1}^{2}+\mathrm{h}_{1}^{2}+\mathrm{C}_{1}^{2}, \\
& \xi_{3}=2 \mathrm{mAf}_{1}^{2}+2 \mathrm{~m} \mathrm{C}_{1}^{2} \mathrm{f}_{1}^{2}+\mathrm{E}_{1}^{2} \\
& \xi_{4}=\mathrm{Ah}_{1}^{2}+\mathrm{B}^{2} \mathrm{~g}_{1}^{2}+\mathrm{AC}_{1}^{2}+\mathrm{C}_{1}^{2} \mathrm{~h}_{1}^{2}+2 \mathrm{~m}^{2} E_{1}^{2} \mathrm{f}_{1}^{2}+\mathrm{BC}_{1}^{2} \mathrm{~g}_{1}^{2}, \\
& \xi_{5}=2 \mathrm{mAC}_{1}^{2} \mathrm{f}_{1}^{2}+\mathrm{AE}_{1}^{2}+\mathrm{BE}_{1}^{2} \mathrm{~g}_{1}^{2}+\mathrm{E}_{1}^{2} \mathrm{~h}_{1}^{2}+2 \mathrm{mf}_{1}^{2} \mathrm{~F}_{1}^{2}, \\
& \xi_{6}=\mathrm{AC}_{1}^{2} \mathrm{~h}_{1}^{2}-\alpha^{2} \mathrm{BC}_{1}^{2} \mathrm{~g}_{1}^{2}+2 \mathrm{Am} E_{1}^{2} \mathrm{f}_{1}{ }^{2}+\mathrm{F}_{1}{ }^{2}\left(\mathrm{~A}+\mathrm{B} \mathrm{g}_{1}{ }^{2}+\mathrm{h}_{1}{ }^{2}\right)-\mathrm{i} \alpha\left(\mathrm{K}_{1}{ }^{2} \mathrm{f}_{1}{ }^{2}+\mathrm{gK}_{1}{ }^{2}\right) \\
& \xi_{7}=\mathrm{AE}_{1}^{2} \mathrm{~h}_{1}^{2}-\mathrm{B}^{2} \mathrm{E}_{1}^{2} \mathrm{~g}_{1}^{2}+2 \mathrm{Am} F_{1}^{2} \mathrm{f}_{1}^{2} \text {, } \\
& \xi_{8}=\left(\mathrm{Ah}_{1}^{2}-\mathrm{B}^{2} \mathrm{~g}_{1}^{2}\right) F_{1}^{2}-\mathrm{i} \alpha \mathrm{K}_{1}^{2} \mathrm{f}_{1}^{2}\left(\mathrm{Am} \mathrm{f}_{1}^{2}+\mathrm{g}\right) \text {. } \\
& \lambda^{\prime 8}+\xi_{1}^{\prime} \lambda^{17}+\xi_{2}^{\prime} \lambda^{\prime 6}+\xi_{3}^{\prime} \lambda^{\prime 5}+\xi_{4}^{\prime} \lambda^{4}+\xi_{5}^{\prime} \lambda^{\prime 3}+\xi_{6}^{\prime} \lambda^{\prime 2}+\xi_{7}^{\prime} \lambda^{\prime}+\xi^{\prime} 8=0
\end{aligned}
$$

where,

$$
\begin{aligned}
& \xi_{1}^{\prime}=2 \mathrm{~m} \mathrm{f}_{1}{ }^{2}, \xi_{2}=\mathrm{A}^{\prime}+\mathrm{B}^{\prime} \mathrm{g}_{1}{ }^{2}+\mathrm{h}_{1}{ }_{1}^{2}+\mathrm{C}_{1}{ }^{2}, \\
& \xi_{3}^{\prime}=2 \mathrm{~mA}^{\prime} \mathrm{f}_{1}{ }^{2}+2 \mathrm{~m} \mathrm{C}_{1}{ }^{2} \mathrm{f}_{1}{ }^{2}+\mathrm{E}_{1}^{\prime}{ }^{2}
\end{aligned}
$$

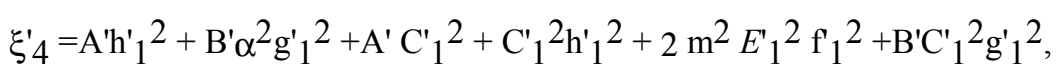

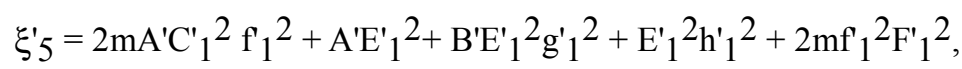




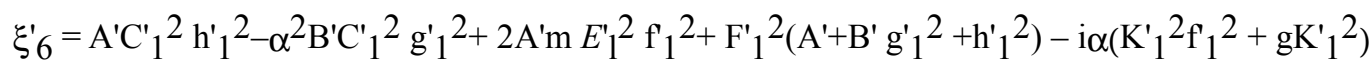

$$
\begin{aligned}
& \xi_{7}^{\prime}=\mathrm{A}^{\prime} \mathrm{E}_{1}^{\prime}{ }^{2} \mathrm{~h}_{1}{ }^{2}-\mathrm{B}^{\prime} \alpha^{2} \mathrm{E}_{1}^{\prime}{ }^{2} \mathrm{~g}_{1}^{\prime}{ }^{2}+2 \mathrm{~A}^{\prime} \mathrm{m} F_{1}^{\prime}{ }_{1}^{2} \mathrm{f}_{1}^{2}, \\
& \xi_{8}^{\prime}=\left(\mathrm{A}^{\prime} \mathrm{h}_{1}^{\prime}{ }^{2}-\mathrm{B}^{\prime} \alpha^{2} \mathrm{~g}_{1}{ }^{2}\right) F_{1}{ }^{2}-\mathrm{i} \alpha \mathrm{K}_{1}^{\prime}{ }_{1}^{2} \mathrm{f}_{1}{ }^{2}\left(\mathrm{~A}^{\prime} \mathrm{m} \mathrm{f}^{\prime}{ }_{1}^{2}+\mathrm{g}\right) \text {. }
\end{aligned}
$$

Where the symbol used in eqns. (21) and (23) are given by eqns. (16) and (18). The constants $A_{j}, B_{j}, C_{j}, D_{j}(j=1,2$, $3,4)$ are related with $A_{j}^{\prime}, B_{j}^{\prime}, C_{j}^{\prime}, D_{j}^{\prime}(j=1,2,3,4)$ in Eq. (19a) and Eq. (19b) by means of first equations in Eq. (15) and Eq. (17).

Equating the coefficients of $e^{-\lambda_{1} z}, e^{-\lambda_{2} z}, e^{-\lambda_{3} z}, e^{-\lambda_{1}^{\prime} z}, e^{-\lambda_{2}^{\prime} z}, e^{-\lambda_{3}^{\prime} z}, e^{-\lambda_{4}^{\prime} z}$ to zero, after substituting Eq. (19a) and Eq. (19b) in the first and 3rd equations of Eq. (15) and Eq. (17) respectively, we get

$$
A_{2}=\gamma_{1} A_{1}, B_{2}=\gamma_{2} B_{1}, C_{2}=\gamma_{3} C_{1}, D_{2}=\gamma_{4} D_{1}
$$

and

$$
\mathrm{A}_{3}=\delta_{1} \mathrm{~A}_{1}, \mathrm{~B}_{3}=\delta_{2} \mathrm{~B}_{1}, \mathrm{C}_{3}=\delta_{3} \mathrm{C}_{1}, \mathrm{D}_{4}=\delta_{4} \mathrm{D}_{4}
$$

where,

$$
\begin{gathered}
\gamma_{\mathrm{j}}=\frac{-K_{1}^{2}}{\lambda_{j}^{4}+C_{1}^{2} \lambda_{j}^{2}-E_{1}^{2} \lambda_{j}+F_{1}^{2}} \quad(\mathrm{j}=1,2,3,4), \\
\delta_{\mathrm{j}}=\frac{1}{g_{1}^{2}}\left[\lambda_{\mathrm{j}}^{2}-2 \mathrm{mf}_{1}^{2} \lambda_{\mathrm{j}}+\mathrm{h}_{1}^{2}+\mathrm{i}^{2} \mathrm{~m} \mathrm{f}_{1}^{2} \gamma_{\mathrm{j}}\right], \\
\mathrm{j}=1,2,3,4 .
\end{gathered}
$$

Similar result holds for medium $\mathrm{M}_{2}$ and usual symbols replacing by dashes respectively.

\section{Boundary Conditions}

(i) The displacement components, temperature and temperature flux at the boundary surface between the media $\mathrm{M}_{1}$ and $\mathrm{M}_{2}$ must be continuous at all times and positions.

$$
\begin{aligned}
\text { i.e. } & {\left[u, v, w, T, p \frac{\partial T}{\partial z}, \frac{\partial T}{\partial z}+h T\right]_{M_{1}}=} \\
& {\left[u, v, w, T, p^{\prime} \frac{\partial T}{\partial z}, \frac{\partial T}{\partial z}+h T\right]_{M_{2}} }
\end{aligned}
$$

(ii) The stress components $\tau_{31}, \tau_{32}, \tau_{33}$ must be continuous at the boundary $\mathrm{z}=0$.

$$
\text { i.e. }\left[\tau_{31}, \tau_{32}, \tau_{33}, \mu_{32}\right]_{M_{1}}=\left[\tau_{31}, \tau_{32}, \tau_{33}, \mu_{32}\right]_{M_{2}}
$$

at $\mathrm{z}=0$ respectively

Where,

$$
\begin{gathered}
\tau_{31}=D_{\mu}\left(2 \frac{\partial^{2} \phi}{\partial x \partial z}+\frac{\partial^{2} \psi}{\partial x^{2}}-\frac{\partial^{2} \psi}{\partial z^{2}}\right)+D_{q} \nabla^{4} \psi \\
\tau_{32}=D_{\mu} \frac{\partial \nu}{\partial z} \\
\mu_{32}=-2 D_{q} \nabla^{2}\left(\frac{\partial \psi}{\partial z}\right), \\
\tau_{33}=D_{\lambda} \nabla^{2} \phi+2 D_{\mu}\left(\frac{\partial^{2} \phi}{\partial z^{2}}+\frac{\partial^{2} \phi}{\partial x \partial z}\right) . \\
-D_{B} T+D_{\mathrm{m}_{e}} H_{0}^{2} \nabla^{2} \phi
\end{gathered}
$$

Applying the boundary conditions, we get

$$
\begin{gathered}
\mathrm{A}_{1}\left(1-\mathrm{i} \gamma_{1} \zeta_{1}\right)+\mathrm{B}_{1}\left(1-\mathrm{i} \gamma_{2} \zeta_{2}\right)+\mathrm{C}_{1}\left(1-\mathrm{i} \gamma_{3} \zeta_{3}\right)+\mathrm{D}_{1}\left(1-\mathrm{i} \gamma_{4} \zeta_{4}\right)-\mathrm{A}_{1}^{\prime}\left(1-\mathrm{i} \gamma_{1}^{\prime} \zeta_{1}^{\prime}\right) \\
\mathrm{B}_{1}^{\prime}\left(1-\mathrm{i} \gamma_{2}^{\prime} \zeta_{2}^{\prime}\right)-\mathrm{C}_{1}^{\prime}\left(1-\mathrm{i} \gamma_{3}^{\prime} \zeta_{3}^{\prime}\right)-\mathrm{D}_{1}^{\prime}{ }_{1}\left(1-\mathrm{i} \gamma_{4}^{\prime} \zeta_{4}^{\prime}\right)=0 \\
\mathrm{C}=\mathrm{C}^{\prime}
\end{gathered}
$$

$\mathrm{p} \lambda_{1} \delta_{1} \mathrm{~A}_{1}+\mathrm{p} \lambda_{2} \delta_{2} \mathrm{~B}_{1}+\mathrm{p} \lambda_{3} \delta_{3} \mathrm{C}_{1}+\mathrm{p} \lambda_{4} \delta_{4} \mathrm{D}_{1}-\mathrm{p}^{\prime} \lambda_{1}^{\prime} \delta_{1}{ }_{1} \mathrm{~A}_{1}^{\prime}+\mathrm{p}^{\prime} \lambda_{2}{ }_{2} \delta_{2} \mathrm{~B}_{1}-\mathrm{p}^{\prime} \lambda_{3}^{\prime} \delta_{3}{ }_{3} \mathrm{C}_{1}^{\prime}-\mathrm{p}^{\prime} \lambda^{\prime}{ }_{4} \delta_{4} \mathrm{C}_{1}{ }_{1}=0$

$$
\begin{gathered}
\left(\lambda_{1}-\mathrm{h}\right) \delta_{1} \mathrm{~A}_{1}+\left(\lambda_{2}-\mathrm{h}\right) \delta_{2} \mathrm{~B}_{1}+\left(\lambda_{3}-\mathrm{h}\right) \delta_{3} \mathrm{C}_{1}+\left(\lambda_{4}-\mathrm{h}\right) \delta_{4} \mathrm{D}_{1}=\left(\lambda_{1}{ }_{1}-\mathrm{h}^{\prime}\right) \delta_{1}{ }_{1} \mathrm{~A}_{1}{ }_{1} \\
+\left(\lambda_{2}^{\prime}-\mathrm{h}^{\prime}\right) \delta_{2}{ }_{2} \mathrm{~B}^{\prime}{ }_{1}+\left(\lambda^{\prime}{ }_{3}-\mathrm{h}^{\prime}\right) \delta^{\prime}{ }_{3} \mathrm{C}^{\prime}{ }_{1}+\left(\lambda_{4}^{\prime}{ }_{4}-\mathrm{h}^{\prime}\right) \delta_{4}{ }_{4} \mathrm{D}^{\prime}{ }_{1}
\end{gathered}
$$


$\left[\mu_{K}^{*}\left(2 \mathrm{i} \zeta_{1}+\gamma_{1}+\zeta_{1}^{2} \gamma_{1}\right)-\eta_{K}^{*} \alpha^{2}\left(\zeta_{1}^{2}-\alpha^{2}\right) \gamma_{1}^{2}\right] \mathrm{A}_{1}+\left[\mu_{K}^{*}\left(2 \mathrm{i} \zeta_{2}+\gamma_{2}+\zeta_{2}^{2} \gamma_{2}\right)-\eta_{K}^{*} \alpha^{2}\left(\zeta_{2}^{2}-1\right) \gamma_{2}\right] \mathrm{B}_{1}+\left[\mu_{K}^{*}(2 \mathrm{i}\right.$ $\left.\left.\zeta_{3}+\gamma_{3}+\zeta_{3}^{2} \gamma_{3}\right)-\eta_{K}^{*} \alpha^{2}\left(\zeta_{3}{ }^{2}-1\right) \gamma_{3}\right] \mathrm{C}_{1}+\left[\mu_{K}^{*}\left(2 \mathrm{i} \zeta_{4}+\gamma_{4}+\zeta_{4}{ }^{2} \gamma_{4}\right)-\eta_{K}^{*} \alpha^{2}\left(\zeta_{4}{ }^{2}-1\right) \gamma_{4}\right] \mathrm{D}_{1}=\left[\mu_{K}^{\prime *}\left(2 \mathrm{i} \zeta_{1}^{\prime}+\gamma_{1}^{\prime}+\right.\right.$

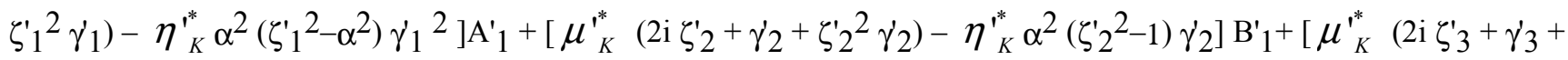
$\left.\left.\zeta_{3}^{\prime}{ }^{2} \gamma_{3}^{\prime}\right)-\eta_{K}^{\prime^{*}} \alpha^{2}\left(\zeta_{3}^{\prime}{ }^{2}-1\right) \gamma_{3}^{\prime}\right] \mathrm{C}_{1}^{\prime}+\left[\mu_{K}^{\prime *}\left(2 \mathrm{i} \zeta_{4}^{\prime}+\gamma_{4}^{\prime}+\zeta_{4}^{\prime}{ }^{2} \gamma_{4}^{\prime}\right)-\eta_{K}^{\prime^{*}} \alpha^{2}\left(\zeta_{4}^{\prime}{ }^{2}-1\right) \gamma_{4}^{\prime}\right] \mathrm{D}_{1}^{\prime}$

$$
\mu_{K}^{*}\left[-\lambda_{4} \mathrm{C}\right]=\mu_{K}^{\prime *}\left[-\lambda_{4}^{\prime} \mathrm{C}^{\prime}\right]
$$

$\mathrm{A}_{1}\left[\left(1_{K}^{*}+\left(\mu_{e}\right)_{K}^{*} H_{0}^{2}\right)\left(\zeta_{1}^{2}-1\right)+2 \mu_{K}^{*}\left(\zeta_{1}^{2}-\mathrm{i} \zeta_{1}\right)-\beta_{K}^{*} \delta_{1}\right]+\mathrm{B}_{1}\left[\left(1_{K}^{*}+\left(\mu_{e}\right)_{K}^{*} H_{0}^{2}\right)\left(\zeta_{2}^{2}-1\right)+2 \mu_{K}^{*}\left(\zeta_{2}^{2}-\mathrm{i} \zeta_{2}\right)-\right.$ $\left.\beta_{K}^{*} \delta_{2}\right]+\mathrm{C}_{1}\left[\left(1_{K}^{*}+\left(\mu_{e}\right)^{*}{ }_{K} H_{0}^{2}\right)\left(\zeta_{3}{ }^{2}-1\right)+2 \mu_{K}^{*}\left(\zeta_{3}{ }^{2}-i \zeta_{3}\right)-\beta_{K}^{*} \delta_{3}\right]+\mathrm{D}_{1}\left[\left(1_{K}^{*}+\left(\mu_{e}\right)^{*}{ }_{K} H_{0}^{2}\right)\left(\zeta_{4}{ }^{2}-1\right)+2 \mu_{K}^{*}\left(\zeta_{4}{ }^{2}-\right.\right.$

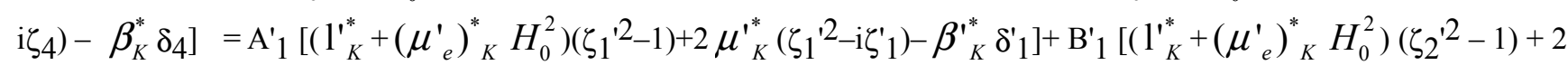
$\left.\mu_{K}^{\prime *}\left(\zeta_{2}^{\prime 2}-i \zeta_{2}^{\prime}\right)-\beta_{K}^{\prime *} \delta_{2}^{\prime}\right]+\mathrm{C}_{1}^{\prime}\left[\left(1_{K}^{\prime *}+\left(\mu_{e}^{\prime}\right)_{K}^{*} H_{0}^{2}\right)\left(\zeta_{3}{ }^{2}-1\right)+2 \mu_{K}^{\prime *}\left(\zeta_{3}{ }^{2}-\mathrm{i} \zeta_{3}^{\prime}\right)-\beta_{K}^{\prime *} \delta_{3}^{\prime}\right]+\mathrm{D}_{1}^{\prime}\left[\left(1_{K}^{*}+\left(\mu_{e}^{\prime}\right)_{K}^{*}\right.\right.$

$$
\left.\left.H_{0}^{2}\right)\left(\zeta_{4}^{\prime 2}-1\right)+2 \mu_{K}^{\prime *}\left(\zeta_{4}^{\prime 2}-i \zeta_{4}^{\prime}\right)-\beta_{K}^{\prime *} \delta_{4}^{\prime}\right]
$$

$\mathrm{A}_{1}\left[\left(\lambda_{K}^{*}\left(\zeta_{1}^{2}-1\right)+2 \mu_{K}^{*}\left(\zeta_{1}^{2}-\mathrm{i} \zeta_{1}\right)-\beta_{K}^{*} \delta_{1}\right]+\mathrm{B}_{1}\left[\lambda_{K}^{*}\left(\zeta_{2}^{2}-1\right)+2 \mu_{K}^{*}\left(\zeta_{2}^{2}-\mathrm{i} \zeta_{2}\right)-\beta_{K}^{*} \delta_{2}\right]+\mathrm{C}_{1}\left[\lambda_{K}^{*}\left(\zeta_{3}^{2}-1\right)+2\right.\right.$ $\left.\mu_{K}^{*}\left(\zeta_{3}^{2}-i \zeta_{3}\right)-\beta_{K}^{*} \delta_{3}\right]+\mathrm{D}_{1}\left[\lambda_{K}^{*}\left(\zeta_{4}{ }^{2}-1\right)+2 \mu_{K}^{*}\left(\zeta_{4}{ }^{2}-i \zeta_{4}\right)-\beta_{K}^{*} \delta_{4}\right]=\mathrm{A}_{1}^{\prime}\left[\lambda_{K}^{\prime *}\left(\zeta_{1}{ }^{2}-1\right)+2 \mu_{K}^{\prime *}\left(\zeta_{1}{ }^{2}-i \zeta_{1}{ }_{1}\right)-\right.$ $\left.\beta_{K}^{\prime *} \delta_{1}^{\prime}\right]_{+} \mathrm{B}_{1}^{\prime}\left[\lambda^{\prime *}\left(\zeta_{2}^{\prime 2}-1\right)+2 \mu_{K}^{\prime *}\left(\zeta_{2}^{\prime 2}-i \zeta_{2}^{\prime}\right)-\beta_{K}^{\prime *} \delta_{2}^{\prime}\right]+\mathrm{C}_{1}^{\prime}\left[\lambda_{K}^{\prime *}\left(\zeta_{3}{ }^{2}-1\right)+2 \mu_{K}^{\prime *}\left(\zeta_{3}^{\prime 2}-i \zeta_{3}^{\prime}\right)-\beta_{K}^{\prime *} \delta_{3}^{\prime}\right]+$

$$
\mathrm{D}_{1}^{\prime}\left[\lambda_{K}^{\prime *}\left(\zeta_{4}^{\prime 2}-1\right)+2 \mu_{K}^{\prime *}\left(\zeta_{4}^{\prime 2}-i \zeta_{4}^{\prime}\right)-\beta_{K}^{\prime^{*}} \delta_{4}^{\prime}\right]
$$
$\eta_{K}^{*}\left[\lambda_{1} \alpha^{2}\left(1-\zeta_{1}{ }^{2}\right) \gamma_{1} \mathrm{~A}_{1}+\lambda_{2} \alpha^{2}\left(1-\zeta_{2}{ }^{2}\right) \gamma_{2} \mathrm{~B}_{1}+\lambda_{3} \alpha^{2}\left(1-\zeta_{3}{ }^{2}\right) \gamma_{3} \mathrm{C}_{1}+\lambda_{4} \alpha^{2}\left(1-\zeta_{4}{ }^{2}\right) \gamma_{4} \mathrm{D}_{1}\right]=\eta_{K}^{\prime *}\left[\lambda_{1}^{\prime} \alpha^{2}\left(1-\zeta_{1}{ }^{2}\right)\right.$
$\left.\gamma_{1} \mathrm{~A}^{\prime}{ }_{1}+\lambda^{\prime}{ }_{2} \alpha^{2}\left(1-\zeta_{2}{ }^{\prime 2}\right) \gamma_{2}{ }^{\prime} \mathrm{B}^{\prime}{ }_{1}+\lambda^{\prime}{ }_{3} \alpha^{2}\left(1-\zeta_{3}{ }^{2}\right) \gamma_{3} \mathrm{C}^{\prime}{ }_{1}+\lambda^{\prime}{ }_{4} \alpha^{2}\left(1-\zeta_{4}{ }^{\prime 2}\right) \gamma_{4}{ }_{4} \mathrm{D}_{1}{ }_{1}\right]$

where, $\zeta_{\mathrm{j}}=\frac{\lambda_{j}}{\alpha}, \zeta_{\mathrm{j}}^{\prime}=\frac{\lambda_{{ }_{j}}{ }^{\alpha}}{\alpha}, \mathrm{j}=1,2,3,4$

and

$$
\begin{gathered}
\lambda_{\mathrm{K}}^{*}=\sum_{K=0}^{n} \lambda_{K}(-i \alpha c)^{K}, \mu_{K}^{*}=\sum_{K=0}^{n} \mu_{K}(-i \alpha c)^{K}, \beta_{K}^{*}=\sum_{K=0}^{n} \beta_{K}(-i \alpha c)^{K}, \\
\eta_{K}^{*}=\sum_{K=0}^{n} \eta_{K}(-i \alpha c)^{K},\left(\mu_{e}\right)_{K}^{*}=\sum_{K=0}^{n}\left(\mu_{e}\right)_{K}(-i \alpha c)^{K}, 1_{K}^{*}=\sum_{K=0}^{n} 1_{K}(-i \alpha c)^{K} \\
\lambda^{\prime} K=\sum_{K=0}^{n} \lambda^{\prime}{ }_{K}(-i \alpha c)^{K}, \mu^{\prime *}=\sum_{K=0}^{n} \mu_{K}^{\prime}(-i \alpha c)^{K}, \beta^{\prime^{*}}=\sum_{K=0}^{n} \beta_{K}^{\prime}(-i \alpha c)^{K}, \\
\eta{ }^{*_{K}^{*}}=\sum_{K=0}^{n} \eta^{\prime}{ }_{K}(-i \alpha c)^{K}, 1^{*}{ }_{K}^{*}=\sum_{K=0}^{n} 1^{\prime}{ }_{K}(-i \alpha c)^{K},\left(\mu_{e}^{\prime}\right)^{*}{ }_{K}^{*}=\sum_{K=0}^{n}\left(\mu_{e}^{\prime}\right)_{K}(-i \alpha c)^{K}
\end{gathered}
$$

From Eq. (26b) and Eq. (26h), we have $\mathrm{C}=\mathrm{C}^{\prime}=0$. Thus there is no propagation of displacement v. Hence SH-waves do not occur in this case.

Finally, eliminating the constants $\mathrm{A}_{1}, \mathrm{~B}_{1}, \mathrm{C}_{1}, \mathrm{D}_{1}, \mathrm{~A}_{1}$, $\mathrm{B}_{1}{ }_{1}, \mathrm{C}_{1}{ }_{1}, \mathrm{D}_{1}{ }_{1}$ from the remaining equations, we get

$$
\operatorname{det}\left(a_{i j}\right)=0, i, j=1,2,3,4,5,6,7,8,9 .
$$

Where,

$$
\begin{gathered}
\mathrm{a}_{11}=1-\mathrm{i} \gamma_{1} \zeta_{1}, \mathrm{a}_{12}=1-\mathrm{i} \gamma_{2} \zeta_{2}, \\
\mathrm{a}_{13}=1-\mathrm{i} \gamma_{3} \zeta_{3}, \mathrm{a}_{14}=1-\mathrm{i} \gamma_{4} \zeta_{4},
\end{gathered}
$$

$$
\begin{gathered}
\mathrm{a}_{15}=\left(\mathrm{i} \gamma_{1}^{\prime} \zeta_{1}^{\prime}-1\right), \mathrm{a}_{16}=\left(\mathrm{i} \gamma_{2}^{\prime} \zeta_{2}^{\prime}-1\right), \\
\mathrm{a}_{17}=\left(\mathrm{i} \gamma_{3}^{\prime} \zeta_{3}^{\prime}-1\right), \mathrm{a}_{18}=\left(\mathrm{i} \gamma_{4}^{\prime} \zeta_{4}^{\prime}-1\right), \\
\mathrm{a}_{21}=\gamma_{1}+\mathrm{i} \zeta_{1}, \mathrm{a}_{22}=\gamma_{2}+i \zeta_{2}, \\
\mathrm{a}_{23}=\gamma_{3}+\mathrm{i} \zeta_{3}, \mathrm{a}_{24}=\gamma_{4}+i \zeta_{4}, \\
\mathrm{a}_{25}=\left(\gamma_{1}^{\prime}+\mathrm{i} \zeta_{1}^{\prime}\right), \mathrm{a}_{26}=\left(\gamma_{2}^{\prime}+i \zeta_{2}^{\prime}\right), \\
\mathrm{a}_{27}=\left(\gamma_{3}^{\prime}+\mathrm{i} \zeta_{3}^{\prime}\right), \mathrm{a}_{28}=\left(\gamma_{4}^{\prime}+i \zeta_{4}^{\prime}\right), \\
\mathrm{a}_{31}=\delta_{1}, \mathrm{a}_{32}=\delta_{2},
\end{gathered}
$$




$$
\begin{aligned}
& a_{33}=\delta_{3}, a_{34}=\delta_{4}, \\
& \mathrm{a}_{35}=-\delta_{1}^{\prime}, \mathrm{a}_{36}=-\delta_{2}^{\prime}, \\
& \mathrm{a}_{37}=-\delta_{3}^{\prime}, \quad \mathrm{a}_{38}=-\delta_{4}^{\prime}, \\
& \mathrm{a}_{41}=\mathrm{p} \lambda_{1} \delta_{1}, \\
& \mathrm{a}_{42}=\mathrm{p} \lambda_{2} \delta_{2}, \mathrm{a}_{43}=\mathrm{p} \lambda_{3} \delta_{3}, \\
& \mathrm{a}_{44}=\mathrm{p} \lambda_{4} \delta_{4}, \mathrm{a}_{45}=-\mathrm{p}^{\prime} \lambda_{1}^{\prime} \delta^{\prime}{ }_{1}, \\
& \mathrm{a}_{46}=-\mathrm{p}^{\prime} \lambda_{2}^{\prime} \delta_{2}^{\prime}, \mathrm{a}_{47}=-\mathrm{p}^{\prime} \lambda_{3}^{\prime} \delta_{3}^{\prime}, \\
& \mathrm{a}_{48}=-\mathrm{p}^{\prime} \lambda_{4}^{\prime} \delta^{\prime}{ }_{4}, \mathrm{a}_{51}=\left(\lambda_{1}-\mathrm{h}\right) \delta_{1}, \\
& a_{52}=\left(\lambda_{2}-h\right) \delta_{2}, a_{53}=\left(\lambda_{3}-h\right) \delta_{3}, \\
& a_{54}=\left(\lambda_{4}-\mathrm{h}\right) \delta_{4}, a_{55}=\left(\lambda^{\prime} 1^{-h^{\prime}}\right) \delta_{1}^{\prime} \\
& a_{56}=\left(\lambda_{2}^{\prime}-h^{\prime}\right) \delta_{2}^{\prime}, a_{57}=\left(\lambda_{3}^{\prime}-h^{\prime}\right) \delta_{3}^{\prime}, \\
& \mathrm{a}_{58}=\left(\lambda^{\prime} 4^{\left.-\mathrm{h}^{\prime}\right)} \delta^{\prime} 4,\right. \\
& \mathrm{a}_{61}=\left[\left(1_{K}^{*}+\left(\mu_{e}\right)_{K}^{*} H_{0}^{2}\right)\left(\zeta_{1}^{2}-1\right)\right. \\
& \left.+2 \mu_{K}^{*}\left(\zeta_{1}^{2}-i \zeta_{1}\right)-\beta_{K}^{*} \delta_{1}\right], \\
& \mathrm{a}_{62}=\left[\left(1_{K}^{*}+\left(\mu_{e}\right)_{K}^{*} H_{0}^{2}\right)\left(\zeta_{2}^{2}-1\right)\right. \\
& \left.+2 \mu_{K}^{*}\left(\zeta_{2}^{2}-i \zeta_{2}\right)-\beta_{K}^{*} \delta_{2}\right], \\
& \mathrm{a}_{63}=\left[\left(1_{K}^{*}+\left(\mu_{e}\right)_{K}^{*} H_{0}^{2}\right)\left(\zeta_{3}^{2}-1\right)\right. \\
& \left.+2 \mu_{K}^{*}\left(\zeta_{3}^{2}-i \zeta_{3}\right)-\beta_{K}^{*} \delta_{3}\right], \\
& \mathrm{a}_{64}=\left[\mu_{K}^{*}\left(2 \mathrm{i} \zeta_{4}+\gamma_{4}+\zeta_{4}{ }^{2} \gamma_{4}\right)-\eta_{K}^{*} \alpha^{2}\left(\zeta_{4}{ }^{2}-1\right) \gamma_{4}\right] \text {, } \\
& \mathrm{a}_{65}=\left[\mu_{K}^{\prime *}\left(2 \mathrm{i} \zeta_{1}^{\prime}+\gamma_{1}^{\prime}+\zeta_{1}^{\prime}{ }_{1}^{2} \gamma_{1}^{\prime}\right)\right. \\
& \left.-\eta_{K}^{\prime *} \alpha^{2}\left(\zeta_{1}^{\prime}{ }^{2}-\alpha^{2}\right) \gamma_{1}^{\prime}{ }^{2}\right] \text {, } \\
& \mathrm{a}_{66}=\left[\left(1_{K}^{\prime *}+\left(\mu_{e}^{\prime}\right)^{*}{ }_{K} H_{0}^{2}\right)\left(\zeta_{2}^{\prime 2}-1\right)\right. \\
& \left.+2 \mu_{K}^{\prime *}\left(\zeta_{2}^{\prime 2}-i \zeta_{2}^{\prime}\right)-\beta_{K}^{\prime *} \delta_{2}^{\prime}\right], \\
& \mathrm{a}_{67}=\left[\left(1_{K}^{\prime *}+\left(\mu_{e}^{\prime}\right)_{K}^{*} H_{0}^{2}\right)\left(\zeta_{3}{ }^{2{ }_{-1}}\right)\right. \\
& \left.+2 \mu_{K}^{\prime *}\left(\zeta_{3}^{\prime 2}-i \zeta_{3}^{\prime}\right)-\beta_{K}^{\prime^{*}} \delta_{3}^{\prime}\right], \\
& \mathrm{a}_{68}=\left[\left(1_{K}^{*}+\left(\mu_{e}^{\prime}\right)^{*}{ }_{K} H_{0}^{2}\right)\left(\zeta_{4}{ }^{2}-1\right)\right. \\
& \left.+2 \mu_{K}^{\prime *}\left(\zeta_{4}^{\prime 2}-i \zeta_{4}^{\prime}\right)-\beta_{K}^{\prime *} \delta_{4}^{\prime}\right],
\end{aligned}
$$

$$
\begin{aligned}
& \mathrm{a}_{71}=\left[\left(\lambda_{K}^{*}\left(\zeta_{1}^{2}-1\right)+2 \mu_{K}^{*}\left(\zeta_{1}^{2}-\mathrm{i} \zeta_{1}\right)-\beta_{K}^{*} \delta_{1}\right],\right. \\
& \mathrm{a}_{72}=\left[\lambda_{K}^{*}\left(\zeta_{2}^{2}-1\right)+2 \mu_{K}^{*}\left(\zeta_{2}^{2}-i \zeta_{2}\right)-\beta_{K}^{*} \delta_{2}\right] \text {, } \\
& \mathrm{a}_{73}=\left[\lambda_{K}^{*}\left(\zeta_{3}^{2}-1\right)+2 \mu_{K}^{*}\left(\zeta_{3}^{2}-i \zeta_{3}\right)-\beta_{K}^{*} \delta_{3}\right],
\end{aligned}
$$

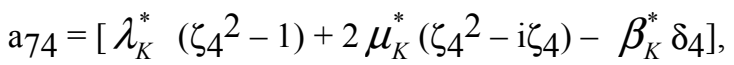

$$
\begin{aligned}
& \mathrm{a}_{75}=\left[\lambda^{\prime *}{ }_{K}\left(\zeta_{1}{ }^{2}-1\right)+2 \mu_{K}^{\prime *}\left(\zeta_{1}{ }^{2}-\mathrm{i} \zeta_{1}^{\prime}\right)-\beta^{\prime^{*}}{ }_{K} \delta_{1}^{\prime}\right] \text {, } \\
& \mathrm{a}_{76}=\left[\lambda^{\prime *}\left(\zeta_{2}^{\prime 2}-1\right)+2 \mu_{K}^{\prime *}\left(\zeta_{2}^{\prime 2}-i \zeta_{2}^{\prime}\right)-\beta_{K}^{\prime{ }^{*}} \delta_{2}^{\prime}\right] \text {, }
\end{aligned}
$$

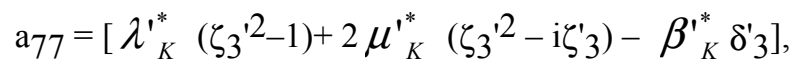

$$
\begin{aligned}
& \mathrm{a}_{78}=\left[\lambda_{K}^{\prime *}\left(\zeta_{4}^{\prime 2}-1\right)+2 \mu_{K}^{\prime *}\left(\zeta_{4}^{\prime 2}-i \zeta_{4}^{\prime}\right)-\beta_{K}^{\prime^{*}} \delta_{4}^{\prime}\right] \text {, } \\
& \mathrm{a}_{81}=\left[\left(1_{K}^{*}+\left(\mu_{e}\right)_{K}^{*} H_{0}^{2}\right)\left(\zeta_{1}^{2}-1\right)\right. \\
& \left.+2 \mu_{K}^{*}\left(\zeta_{1}^{2}-i \zeta_{1}\right)-\beta_{K}^{*} \delta_{1}\right] \\
& \mathrm{a}_{82}=\left[\left(1_{K}^{*}+\left(\mu_{e}\right)^{*}{ }_{K} H_{0}^{2}\right)\left(\zeta_{2}{ }^{2}-1\right)\right. \\
& \left.+2 \mu_{K}^{*}\left(\zeta_{2}^{2}-i \zeta_{2}\right)-\beta_{K}^{*} \delta_{2}\right], \\
& \mathrm{a}_{83}=\left[\lambda_{K}^{*}\left(\zeta_{3}^{2}-1\right)+2 \mu_{K}^{*}\left(\zeta_{3}^{2}-i \zeta_{3}\right)-\beta_{K}^{*} \delta_{3}\right], \\
& \mathrm{a}_{84}=\left[\left(1_{K}^{*}+\left(\mu_{e}\right)_{K}^{*} H_{0}^{2}\right)\left(\zeta_{4}{ }^{2}-1\right)\right. \\
& \left.+2 \mu_{K}^{*}\left(\zeta_{4}^{2}-i \zeta_{4}\right)-\beta_{K}^{*} \delta_{4}\right], \\
& \mathrm{a}_{85}=\left[\left(1_{K}^{\prime *}+\left(\mu_{e}^{\prime}\right)_{K}^{*} H_{0}^{2}\right)\left(\zeta_{1}^{\prime 2}{ }_{-1}\right)\right. \\
& \left.+2 \mu_{K}^{\prime *}\left(\zeta_{1}{ }^{2}-\mathrm{i} \zeta_{1}^{\prime}\right)-\beta^{\prime{ }^{*}} \delta_{1}^{\prime}\right], \\
& \mathrm{a}_{86}=\left[\left(1_{K}^{\prime *}+\left(\mu_{e}^{\prime}\right)_{K}^{*} H_{0}^{2}\right)\left(\zeta_{2}^{\prime 2}-1\right)\right. \\
& \left.+2 \mu_{K}^{\prime *}\left(\zeta_{2}^{\prime 2}-i \zeta_{2}^{\prime}\right)-\beta_{K}^{\prime^{*}} \delta_{2}^{\prime}\right], \\
& \mathrm{a}_{87}=\left[\left(1_{K}^{\prime *}+\left(\mu_{e}^{\prime}\right)_{K}^{*} H_{0}^{2}\right)\left(\zeta_{3}{ }^{\prime 2}-1\right)\right. \\
& \left.+2 \mu_{K}^{\prime *}\left(\zeta_{3}^{\prime 2}-i \zeta_{3}^{\prime}\right)-\beta_{K}^{*^{*}} \delta_{3}^{\prime}\right], \\
& { }_{88}=\left[\lambda_{K}^{\prime *}\left(\zeta_{4}^{\prime 2}-1\right)+2 \mu_{K}^{\prime *}\left(\zeta_{4}^{\prime 2}-i \zeta_{4}^{\prime}\right)-\beta_{K}^{\prime{ }^{*}} \delta_{4}^{\prime}\right] \text {, } \\
& \mathrm{a}_{91}=\eta_{K}^{*} \lambda_{1} \alpha^{2}\left(1-\zeta_{1}^{2}\right) \gamma_{1} \\
& \mathrm{a}_{92}=\eta_{K}^{*} \lambda_{2} \alpha^{2}\left(1-\zeta_{2}^{2}\right) \gamma_{2} \\
& \mathrm{a}_{93}=\eta_{K}^{*} \lambda_{3} \alpha^{2}\left(1-\zeta_{3}{ }^{2}\right) \gamma_{3}
\end{aligned}
$$




$$
\begin{gathered}
\mathrm{a}_{94}=\eta_{K}^{*} \lambda_{4} \alpha^{2}\left(1-\zeta_{4}{ }^{2}\right) \gamma_{4} \\
\mathrm{a}_{95}=\eta_{K}^{\prime^{*}}\left[\lambda_{1}^{\prime} \alpha^{2}\left(1-\zeta_{1}{ }^{\prime 2}\right) \gamma_{1}^{\prime}\right. \\
\mathrm{A}_{96}=\eta_{K}^{\prime *} \lambda_{2}^{\prime} \alpha^{2}\left(1-\zeta_{2}^{\prime 2}\right) \gamma_{2}^{\prime} \\
\mathrm{A}_{97}=\eta_{K}^{\prime^{*}} \lambda_{3}^{\prime} \alpha^{2}\left(1-\zeta_{3}^{\prime}{ }^{2}\right) \gamma_{3} \\
\mathrm{a}_{98}=\eta_{K}^{\prime^{*}} \lambda_{{ }_{4}}^{\prime} \alpha^{2}\left(1-\zeta_{4}^{\prime 2}\right) \gamma_{4}^{\prime}
\end{gathered}
$$

From Eq. (27), we obtain velocity of surface waves in common boundary between two viscoelastic, nonhomogeneous solid media under the influence of thermal and magnetic field, where the viscosity is of general nth order involving time rate of change of strain.

\section{Particular Cases}

\subsection{Stoneley Waves}

It is the generalized form of Rayleigh waves in which we assume that waves are propagated along the common boundary of the two semi-infinite media $\mathrm{M}_{1}$ and $\mathrm{M}_{2}$. Thus Eq. (27) determine the wave velocity equation for Stoneley waves in the case of general magneto-thermo viscoelastic, non-homogeneous solid media of nth order involving time

$$
\begin{aligned}
& {\left[\mu_{K}^{*}\left(2 \mathrm{i} \zeta_{1}+\gamma_{1}+\zeta_{1}^{2} \gamma_{1}\right)-\eta_{K}^{*} \alpha^{2}\left(\zeta_{1}^{2}-\alpha^{2}\right) \gamma_{1}{ }^{2}\right] \mathrm{A}_{1}+\left[\mu_{K}^{*}\left(2 \mathrm{i} \zeta_{2}+\gamma_{2}+\zeta_{2}^{2} \gamma_{2}\right)-\eta_{K}^{*} \alpha^{2}\left(\zeta_{2}^{2}-1\right) \gamma_{2}\right] \mathrm{B}_{1}} \\
& +\left[\mu_{K}^{*}\left(2 \mathrm{i} \zeta_{3}+\gamma_{3}+\zeta_{3}^{2} \gamma_{3}\right)-\eta_{K}^{*} \alpha^{2}\left(\zeta_{3}^{2}-1\right) \gamma_{3}\right] \mathrm{C}_{1}+\left[\mu_{K}^{*}\left(2 \mathrm{i} \zeta_{4}+\gamma_{4}+\zeta_{4}^{2} \gamma_{4}\right)-\eta_{K}^{*} \alpha^{2}\left(\zeta_{4}{ }^{2}-1\right) \gamma_{4}\right] \mathrm{D}_{1}=0(29 \mathrm{a}) \\
& \mathrm{A}_{1}\left[\left(1_{K}^{*}+\left(\mu_{e}\right)_{K}^{*} H_{0}^{2}\right)\left(\zeta_{1}^{2}-1\right)+2 \mu_{K}^{*}\left(\zeta_{1}^{2}-\mathrm{i} \zeta_{1}\right)-\beta_{K}^{*} \delta_{1}\right]+\mathrm{B}_{1}\left[\left(1_{K}^{*}+\left(\mu_{e}\right)_{K}^{*} H_{0}^{2}\right)\left(\zeta_{2}^{2}-1\right)+2 \mu_{K}^{*}\left(\zeta_{2}^{2}-\mathrm{i} \zeta_{2}\right)\right. \\
& \left.-\beta_{K}^{*} \delta_{2}\right]+\mathrm{C}_{1}\left[\left(1_{K}^{*}+\left(\mu_{e}\right)_{K}^{*} H_{0}^{2}\right)\left(\zeta_{3}^{2}-1\right)+2 \mu_{K}^{*}\left(\zeta_{3}^{2}-\mathrm{i} \zeta_{3}\right)-\beta_{K}^{*} \delta_{3}\right]+\mathrm{D}_{1}\left[\left(1_{K}^{*}+\left(\mu_{e}\right)_{K}^{*} H_{0}^{2}\right)\left(\zeta_{4}^{2}-1\right)\right. \\
& \left.+2 \mu_{K}^{*}\left(\zeta_{4}^{2}-\mathrm{i} \zeta_{4}\right)-\beta_{K}^{*} \delta_{4}\right]=0
\end{aligned}
$$

$$
\begin{array}{r}
\mathrm{A}_{1}\left[\left(\lambda_{K}^{*}\left(\zeta_{1}^{2}-1\right)+2 \mu_{K}^{*}\left(\zeta_{1}^{2}-\mathrm{i} \zeta_{1}\right)-\beta_{K}^{*} \delta_{1}\right]+\mathrm{B}_{1}\left[\lambda_{K}^{*}\left(\zeta_{2}^{2}-1\right)+2 \mu_{K}^{*}\left(\zeta_{2}^{2}-\mathrm{i} \zeta_{2}\right)-\beta_{K}^{*} \delta_{2}\right]+\mathrm{C}_{1}\left[\lambda_{K}^{*}\left(\zeta_{3}^{2}-1\right)+2\right.\right. \\
\left.\mu_{K}^{*}\left(\zeta_{3}^{2}-\mathrm{i} \zeta_{3}\right)-\beta_{K}^{*} \delta_{3}\right]+\mathrm{D}_{1}\left[\lambda_{K}^{*}\left(\zeta_{4}^{2}-1\right)+2 \mu_{K}^{*}\left(\zeta_{4}^{2}-\mathrm{i} \zeta_{4}\right)-\beta_{K}^{*} \delta_{4}\right]=0
\end{array}
$$

From equation (27), we have

$$
\left(\lambda_{1}-\mathrm{h}\right) \delta_{1} \mathrm{~A}_{1}+\left(\lambda_{2}-\mathrm{h}\right) \delta_{2} \mathrm{~B}_{1}+\left(\lambda_{3}-\mathrm{h}\right) \delta_{3} \mathrm{C}_{1}+\left(\lambda_{4}-\mathrm{h}\right) \delta_{4} \mathrm{D}_{1}=0
$$

Eliminating $A_{1}, B_{1}, C_{1}$ and $D_{1}$ from eqns. (29a), (29b), (29c) and (29d) we get

$$
\operatorname{det}\left(\mathrm{b}_{\mathrm{ij}}\right)=0, \mathrm{i}, \mathrm{j}=1,2,3,4 .
$$

Thus Eq. (30), gives the wave velocity equation for Rayleigh waves in a non-homogeneous, magneto-thermo viscoelastic solid media of nth order involving time rate of strain. From Eq. (30), it is follows that Dispersion equation of Rayleigh waves depends upon the non-homogeneity, the viscous, gravity, magnetic and thermal fields.

This equation, of course, is in complete agreement with the corresponding classical result by Bullen, when the effects of thermal, gravity, magnetic viscous field and nonhomogeneity are absent.

Case-2 Dispersion equation of Rayleigh waves in terms of non-homogeneity, viscous, gravity, couple-stress and thermal fields

Eq. (26f), Eq. (26h) and Eq. (26k) reduces to, 


$$
\begin{gathered}
{\left[\mu_{K}^{*}\left(2 \mathrm{i} \zeta_{1}+\gamma_{1}+\zeta_{1}{ }^{2} \gamma_{1}\right)-\eta_{K}^{*} \alpha^{2}\left(\zeta_{1}{ }^{2}-\alpha^{2}\right) \gamma_{1}{ }^{2}\right] \mathrm{A}_{1}+\left[\mu_{K}^{*}\left(2 \mathrm{i} \zeta_{2}+\gamma_{2}+\zeta_{2}{ }^{2} \gamma_{2}\right)-\eta_{K}^{*} \alpha^{2}\left(\zeta_{2}{ }^{2}-1\right) \gamma_{2}\right] \mathrm{B}_{1}} \\
+\left[\mu_{K}^{*}\left(2 \mathrm{i} \zeta_{3}+\gamma_{3}+\zeta_{3}{ }^{2} \gamma_{3}\right)-\eta_{K}^{*} \alpha^{2}\left(\zeta_{3}{ }^{2}-1\right) \gamma_{3}\right] \mathrm{C}_{1}+\left[\mu_{K}^{*}\left(2 \mathrm{i} \zeta_{4}+\gamma_{4}+\zeta_{4}{ }^{2} \gamma_{4}\right)-\eta_{K}^{*} \alpha^{2}\left(\zeta_{4}{ }^{2}-1\right) \gamma_{4}\right] \mathrm{D}_{1}=0 \\
{\left[\lambda_{1} \alpha^{2}\left(1-\zeta_{1}{ }^{2}\right) \gamma_{1} \mathrm{~A}_{1}+\lambda_{2} \alpha^{2}\left(1-\zeta_{2}{ }^{2}\right) \gamma_{2} \mathrm{~B}_{1}+\lambda_{3} \alpha^{2}\left(1-\zeta_{3}{ }^{2}\right) \gamma_{3} \mathrm{C}_{1}+\lambda_{4} \alpha^{2}\left(1-\zeta_{4}{ }^{2}\right) \gamma_{4} \mathrm{D}_{1}\right]=0}
\end{gathered}
$$

From equation (27), we have

$$
\left(\lambda_{1}-\mathrm{h}\right) \delta_{1} \mathrm{~A}_{1}+\left(\lambda_{2}-\mathrm{h}\right) \delta_{2} \mathrm{~B}_{1}+\left(\lambda_{3}-\mathrm{h}\right) \delta_{3} \mathrm{C}_{1}+\left(\lambda_{4}-\mathrm{h}\right) \delta_{4} \mathrm{D}_{1}=0
$$

Eliminating $\mathrm{A}_{1}, \mathrm{~B}_{1}, \mathrm{C}_{1}$ and $\mathrm{D}_{1}$ from eqns. (31a), (31b), (31c) and (31d), we get

$$
\operatorname{det}\left(\mathcal{E}_{i j}\right)=0, \mathrm{i}, \mathrm{j}=1,2,3,4 \text {. }
$$

Thus Eq. (32), gives the wave velocity equation for Rayleigh waves in a non-homogeneous, thermo viscoelastic solid media of nth order involving time rate of strain. From Eq. (32), it is follows that Dispersion equation of Rayleigh waves depends upon the non-homogeneity, the viscous, gravity, couple-stress and thermal fields.

This equation, of course, is in complete agreement with the corresponding classical result by Bullen, when the effects of thermal, gravity, couple-stress, viscous field and non-homogeneity are absent.

\subsection{Love Waves}

To investigate the possibility of love waves in a nonhomogeneous, viscoelastic solid media, we replace medium $\mathrm{M}_{2}$ is obtained by two horizontal plane surfaces at a distance $\mathrm{H}$-apart, while $\mathrm{M}_{1}$ remains infinite. For medium $\mathrm{M}_{1}$, the displacement component $v$ remains same as in general case given by Eq. (19). For the medium $\mathrm{M}_{2}$, we preserve the full solution, since the displacement component along y-axis i.e. no longer diminishes with increasing distance from the boundary surface of two media.

Thus

$$
\mathrm{v}^{\prime}=C_{1} e^{\lambda_{5}^{\prime} z+i \alpha(x-c t)}+C_{2} e^{-\lambda_{5}^{\prime} z+i \alpha(x-c t)}
$$

In this case, the boundary conditions are

(i) $\mathrm{v}$ and $\tau_{32}$ are continuous at $\mathrm{z}=0$

(ii) $\tau_{32}^{\prime}=0$ at $\mathrm{z}=-\mathrm{H}$.

Applying boundary conditions (i) and (ii) and using Eq. (19) and Eq. (26), we get

$$
\begin{gathered}
\mathrm{C}=\mathrm{C}_{1}+\mathrm{C}_{2} \\
-\mu_{K}^{*} \lambda_{5} \mathrm{C}=\left(\mu_{\mathrm{K}}^{\prime}\right)^{*}\left[\lambda_{5}^{\prime} \mathrm{C}_{1}-\lambda_{5}^{\prime} \mathrm{C}_{2}\right]
\end{gathered}
$$

$$
C_{1} e^{-\lambda_{5}^{\prime} H}-C_{2} e^{\lambda_{5}^{\prime} H}=0
$$

On eliminating the constants $\mathrm{C}, \mathrm{C}_{1}$ and $\mathrm{C}_{2}$ from Eq. (34a), Eq. (34b) and Eq. (34c), we get

$$
\tanh \left(\lambda_{5}^{\prime} \mathrm{H}\right)=-\frac{\lambda_{5} \mu_{K}^{*}}{\lambda_{5}^{\prime}\left(\mu_{K}^{\prime}\right) *} .
$$

Thus equation (35) gives the wave velocity equation for Love waves under the influence of gravity and couple stress parameter in a non-homogeneous, thermo, magneto, viscoelastic elastic solid medium of $\mathrm{n}^{\text {th }}$ order involving time rate of strain. Clearly it depends upon the non-homogeneity of the material medium and viscosity, couple stress parameter and independent of thermal and gravitational fields.

Equation (35), of course is in complete agreement with the corresponding classical result by Chadwick, when the effects of viscous field, non-homogeneity, gravity and couple-stress parameter are absent. Further in the absence of temperature field, this equation is in complete agreement with the corresponding classical result by Bullen.

\section{Conclusions}

The surface waves in a non-homogeneous, isotropic, viscoelastic solid medium under gravity of nth order including time rate of strain are investigated. It is observed that viscoelastic surface waves are affected by the time rate of strain parameters. These parameters influence the wave velocity to an extent depending on the corresponding constants characterizing the magneto thermo and viscoelasticity of the material. So the results of this analysis become useful in circumstances where these effects cannot be neglected. These velocities depend upon the wave number ' $\alpha$ ' confirming that these waves are affected by nonhomogeneity of the material medium.

Love waves do not depend on temperature; these are only affected by viscous, gravity, couple-stress, magnetic fields and non-homogeneity of the material medium. In 
absence of all fields and non-homogeneity, the dispersion equation is in complete agreement with the corresponding classical result.

Rayleigh waves in a non-homogeneous, general magneto-thermo viscoelastic solid medium of higher order including time rate of change of strain we find that the wave velocity equation proves that there is dispersion of waves due to the presence of non-homogeneity, temperature, gravity, couple-stress, magnetic field and viscosity. The results are in complete agreement with the corresponding classical results in the absence of all fields and compression.

The wave velocity equation of Stoneley waves is very similar to the corresponding problem in the classical theory of elasticity. The dispersion of waves is due to the presence of non-homogeneity, gravity, couple-stress, magnetic field, temperature and viscoelasticity of the solid. Also, wave velocity equation of this generalized type of surface waves is in complete agreement with the corresponding classical result in the absence of all fields and non-homogeneity.

The solution of wave velocity equation for Stoneley waves cannot be determined by easy analytical methods however we can apply numerical techniques to solve this determinantal equation by choosing suitable values of physical constants for both media $M_{1}$ and $M_{2}$.

\section{References}

[1] Carcione JM, Wave propagation in anisotropic linear viscoelastic media; theory and simulated wave fields, Geophysical Journal International, 1990, 101, 739-750.

[2] Stoneley R, Proc. R. Soc. A, 806, 1924, 416-28.

[3] Bullen K.E, Theory of Seismology, Cambridge University Press, 1965.

[4] Hunter, SC, Viscoelastic waves, Progress in solid mechanics, I. (Ed: Sneddon IN and Hill R) Cambridge University Press, 1970.

[5] Jeffreys H, The Earth, Cambridge University Press, 1970.

[6] Bromwich TJ, On the influence of gravity on elastic waves, and, in particular, on the vibrations of an elastic globe, Proc. London Math. Soc., 1898, 30, 98-120.

[7] Love AEH, Some problems of Geodynamics. Cambridge University Press, London, 1965.

[8] Sezawa K, Dispersion of elastic waves propagated on the surface of stratified bodies and on curved surfaces, Bull. Earthq. Res. Inst. Tokyo, 1927, 3, 1-18.

[9] Thomson WT, Transmissions of elastic waves through a stratified solid medium, J. Appl. Phys., 1950, 21, 89-93.
[10] Haskell, NA, The dispersion of surface waves in multilayered media, Bull. Seis. Soc. Amer., 1953, 43, 17-34.

[11] Ewing WM, Jardetzky WS, Press F, Elastic waves in layered media, McGraw-Hill, New York, 1957.

[12] M.A. Biot, Mechanics of incremental Deformations, J. Willy, 1965.

[13] Jones JP, Wave propagation in a two layered medium, J. Appl. Mechanics, 1964, 213-222.

[14] De SK, Sengupta PR, Influence of gravity on wave propagation in an elastic layer, J.Acanst. Soc. Am., 1974, 55, 5-13.

[15] Sengupta PR, Acharya D, The influence of gravity on the propagation of waves in a thermoelastic layer, Rev. Romm. Sci. Techmol. Mech. Appl., Tome, 1979, 24, 395-406.

[16] Brunelle EJ, Surface wave propagation under initial tension or compression, Bull. Seismol. Soc. Am., 1973, 63, 18951899.

[17] Roy PP, Wave propagation in a thinly two layered medium with stress couples under initial stresses, Acta Mechanisms, $1984,54,1-21$.

[18] Datta BK, Some observation on interactions of Rayleigh waves in an elastic solid medium with the gravity field, Rev. Roumaine Sci. Tech. Ser. Mec. Appl., 1986, 31, 369-374.

[19] Goda MA, The effect of inhomogeneity and onisotropy on Stoneley waves, Acta Mech., 1992, 93, 89-98.

[20] Abd-Alla AM, Ahmed SM, Rayleigh waves in an orthotropic thermoelastic medium under gravity field and initial stress, J. Earth, Moon Planets, 1996, 75, 185-197.

[21] Kakar R, Kakar S, Gupta KC, Surface wave propagation in non homogeneous, general magneto-thermo, viscoelastic media, International Journal of IT, Engineering and Applied Sciences Research, 2012, 1(1), 42-49.

[22] Kakar R and S. Kakar, Rayleigh waves in a nonhomogeneous granular media. J. Chem. Bio. Phy. Sci. Sec. B, 2012-2013, 3(1),464-509.

[23] Kakar R, Kakar S, Thermoelastic Surface Waves Propagation in Non-Homogeneous Viscoelastic Media of Higher Order. JECET, 2012-2013, 2(1), 57-69.

[24] Kakar R, Kakar S, Magnetoelastic Surface Waves Propagation in Non-Homogeneous Viscoelastic Media of Higher Order. J. Chem. Bio. Phy. Sci. Sec. B, 2012-2013, 3(1), 498478 .

[25] Kakar R, Kakar S, Propagation of Stoneley, Rayleigh and Love waves in viscoelastic media of higher order. International Journal of Current Research, 2012, 4(12), 249-257.

[26] Voigt W, Abh. Gesch. Wiss, 1887, 34. 